\title{
A Novel Shape Signature of Geometric Mean of Segmented Centroid Distance Function to Track the Object through Fourier Descriptors
}

\author{
M. H. Sidram \\ J.S.S. Research Foundation \\ J.S.S. Technical Institutions Campus \\ Mysore, Karnataka, India
}

\author{
Nagappa U. Bhajantri \\ Department of CS\&Engg. \\ Government Engineering College \\ Chamarajanagar
}

\begin{abstract}
The process explicitly dedicated to estimate the path of the object as it moves along the region of scene in the image plane is principle of tracking. In other words it is a strategy to detect and track moving object through a sequence of frames. In this work, optical flow based on Horn-Schunk with Barren, Fleet and Beuchemin (BFB) kernel has been employed to estimate the motion vectors.

The peripheries of moving objects are extracted for different shape signatures such as boundary, edge, area, curvature and centroid distance functions. Fourier descriptors of particular shape signature for each of the candidate and model template are extracted. Similarity between the model and candidate templates is confirmed by corresponding minimum Minkowski distance. Subsequently, best match candidate will be updated.
\end{abstract}

However, centroid distance function has remarked some potential. Thus, it further motivated to mine exhaustively to throw in the proposed novel criteria such as the Geometric Mean of Segmented Centroid (GMSC) distance function to track the object. The proposed method of GMSC distance function has shown significant change in the tracking outcome.

\section{General Terms:}

Object Tracking, Fourier Descriptors.

\section{Keywords:}

Gray Optical flow, Shape signature, Fourier descriptors (FD), Object Tracking, Geometric Mean of Segmented Centroid distance function (GMSC)

\section{INTRODUCTION}

In recent times the broader area of computer vision magnanimously attracted and extended the attention towards the object tracking as major focus of cram. A specific concentration drawing mechanism on the whole is worthwhile to track the object in a scene. In other words, it is also apractice of ascertaining the correspondence to the objects in sequence of frames. Thus majority of applications are in the Robot navigation, Video surveillance, Traffic monitoring etc. There is no dearth of relevant literature in tracking object emerged in a moderate scene. It could be possible through spatial or appearance based model. Secondly several processes are evolved from frequency sphere. There are several approaches [1] [2] [3] for tracking object in a scene that are Point, Kernel and silhouette tracking. Template matching formed with sub-class of Kernel tracking.

In order to trace the interested object, there are strategies available in literature like frame differencing, optical flow etc [4] [5]. In the proposed method, the moving objects are cropped using the well known gray optical flow strategy of Horn-Schunk. This is very responsible criteria to address the computational aspects precisely for determining the velocity vectors related to the motion. The BFB kernel has portrayed the better optical flow density estimation which is able to fabricate the binary pattern of object in a scene.

Subsequently, the obtained binary moving objects are labeled using the connected component presumption. The boundary of them can be extracted using Canny edge detector whose performance is finer. Since obtained information is crispy and requisite to narrate the pattern as other strategies [6] [7] such as algebraic moments, Zernike moments, wavelets and many are required the redundant contents of the object. However, Fourier transforms is one of the approaches which is capable to represent the edge information as coefficients in a dimension.

Fourier coefficients are also addressed as Fourier descriptors (FDs). Discrete Fourier Transforms (DFT) emulates these descriptors with the certain advantages like robust to noise, simple to estimate and compact in size which make it superior for the specific applications. As low frequency coefficients yield the broad and general features of the pattern whereas high frequency coefficients provide detailed features associated with pattern. Astonishingly human perception of pattern and its depiction via Fourier descriptors is identical. The spectrum comprehends the plot of amplitude or angle versus frequency as each of the Fourier descriptors consists of amplitude and angle.

With the rapid growth of many shape representation methods [8] [9] [10] [11] [12] [13] and most of those strategies are attempted to represent the shape information adequately. On the other hand it may be complicated to do normalization which makes matching difficult. Here, Fourier descriptors have portrayed the 
potentiality to curb the above predicament to the larger extent. In fact, the literature glimpses as moment descriptors and other global descriptors have feeble discrimination capability [6], [7], [14], [15], [16]. Similarly, FDs have motivated us to make an effort to enhance the discrimination capability due to its prevailing nature of noise sensitivity in the representation of shape signatures.

Moving objects are obtained by way of Horn-schunk approach and the appropriate threshold selection to extract the velocity vectors is a challenging task. In other words it is contextual and volatile with respect to scene and also sensitive to variant of dataset. Here, this work has extended the experimentation over the benchmark dataset such as PETS2001 and TAXI video clips of outdoor surveillance.

The remaining part of this work is organized into 5 sections. Section 2 presents the comprehension of contemporary literature. Section 3 describes the milestones which are indispensable to identify the research work and emphasized the proposed geometric mean of segmented centroid distance function based shape signature. Subsequently, proposed complete algorithm displayed. In section 4 exhaustive experimental observations and results are revealed. Finally conclusion is completed in Section 5.

\section{RELATED WORK}

The past decade has seen explosive growth with the effort of research community in proposing potential object tracking model in a scene or video. In todays world object tracking information is indispensible in most of important video based activities. In other words, Precise tracking of object information according to the users need is an essential task. A content based image retrieval to detect the object has extended by the author Zhang et al. [8] and they had made an attempt to compare the performance between FDs and Curvature Scale Space(CSS). Eventually, their work had a success in proving the supremacy of Fourier descriptors.

The researcher, Amanatiadis et al. [15] have worked on the content based image retrieval and its comparative study among scale, rotation and translation invariant descriptors.

An extraction of Fourier descriptors of an edge making them invariant to scale, rotation and translation for locating the Logos in the image database are attempted by the author Andre Folkers et al.[2].

There are also some other algorithms that focus on addressing the shape signatures and polygonal approximations by the research team Yang et al. [6]. They have made comparative study of shape related methods in the content based image retrieval context. Sarfraz M.et al. [17] have tried to extract the boundary of objects and created Fourier descriptors. They also discussed the requirement of number of descriptors in object recognition for acceptable identification.

Here, the review of literature done meticulously in the broader area of object tracking and selection of appropriate literature needs to explore potential approach. The features are drawn from Zhang et al. [8], Yang et al. [6] and Sarfraz M. et al. [17] modalities in extracting the Fourier descriptors for various shape signatures. There may be small amount of work has been reported towards the object recognition. However, still there is a huge scope to propose enhancement over existing mathematical modeling through relevant computing strategy for automatic object tracking.

In this direction, it is planned to exploit the capability of FDs technique to track the object with suitably modified shape signature. In other words, papers [6], [8], [17] further motivated to carry out the research on Fourier descriptors with optical flow.

\section{PROPOSED METHODOLOGY}

The essence of mathematical supports involved with the gray optical flow, Fourier Descriptors are glanced in the following sub divisions. Subsequently, the proposed algorithm has been narrated.

\subsection{Gray Optical Flow Estimation}

$$
I(x, y, t)=I(x+\delta x, y+\delta y, t+\delta t)
$$

Here, $x, y$ and $t$ are spatial and temporal primitives respectively. If $\delta t$ is small, then expansion of Eq.(1) using Taylor series confers.

$$
\begin{aligned}
I(x+\delta x, y+\delta y, t+\delta t) & =\frac{\partial I}{\partial x} \delta x+\frac{\partial I}{\partial y} \delta y+\frac{\partial I}{\partial t} \delta t+\ldots \\
I_{x} u+I_{y} v & =-I_{t}
\end{aligned}
$$

The Eq. 3 produces a unsolved equation with two unknowns and this is addressed properly by Horn-Schunck. From these equations iterative scheme is derived and expressed in Eqs. (4) and (5).

$$
\begin{aligned}
u^{n+1} & =\bar{u}^{n}-\frac{I_{x}\left[I_{x} \bar{u}^{n}+I_{y} \bar{v}^{n}+I_{t}\right]}{\left(a^{2}+I_{x}{ }^{2}+I_{y}{ }^{2}\right)} \\
v^{n+1} & =\bar{v}^{n}-\frac{I_{y}\left[I_{x} \bar{u}^{n}+I_{y} \bar{v}^{n}+I_{t}\right]}{\left(a^{2}+I_{x}{ }^{2}+I_{y}{ }^{2}\right)}
\end{aligned}
$$

Using suitable threshold, author attempted to evaluate the optical flow to extract moving objects. In view of unique representation of the pattern signature through the Fourier descriptors is expressed in the following subsection.

\subsection{Extraction of Fourier Descriptors}

The abstract representations of the objects are predominantly deemed through boundary of the moving object. In other words, shape signature of moving object is characterized by appropriate signature. There are various shape signatures noticed in the literature [8], [11] such as position function, edge function, centroid distance, curvature and cumulative angular function etc.

Shape representation in the form of features is more powerful than the features like texture, color etc. There are mainly two types of shape descriptors with equal importance. Firstly contour based and secondly region based shape descriptors [6], [8], [15]. In contour based strategy, boundary information is exploited to create the features and the information of all the pixels is needed in region based method. Here, contour is a boundary of an object representing salient region and correct identification of object is quite possible with its edge information as shown in Figure 1. Literature substantiates the work and Canny edge detector proved to be the best method to extract the edge of an object without noise. Boundary information of objects can be expressed through the Fourier coefficients or descriptors. However, low frequency coefficients yield the broad and general features of the shape whereas high frequency coefficients provide detailed features associated with shape.

FDs are naturally invariant to the translation by discarding first descriptor. Consequently, they are made invariant to the rotation by considering only magnitudes of the coefficients. In other words, the rotation information is embedded in the phase of each coefficient. In the sequence, normalization is effected to make them invariant 


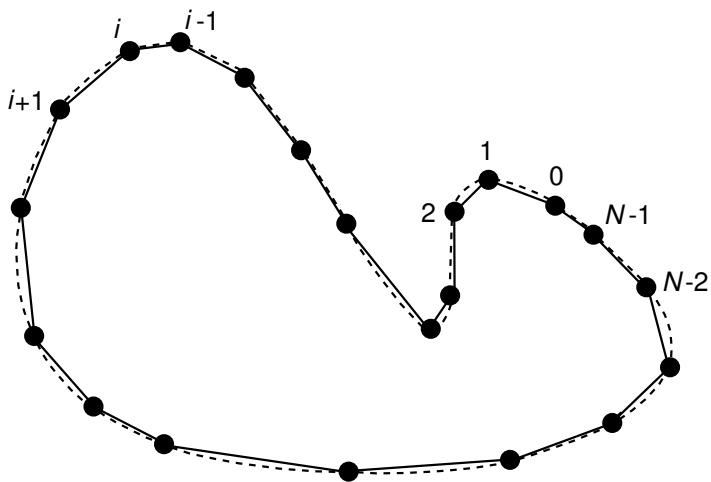

Fig. 1. Shape of an object.

to the scaling by dividing all the Fourier descriptors with second descriptor. But in case of centroid distance function, normalization is done by dividing all the FDs by first descriptor, which is expressed in Eq. 147. Here, the essential invariance to starting point is obtained by subtracting the phase of second Fourier descriptor from phase of all FDs as given in Eq. 15. If the shape information in spatial domain is expressed through real valued then first half of the FDs are sufficient to represent the original shape. Since other half of the descriptors are conjugate values of first half.

$$
u(N)=x(n)+j y(n)
$$

At this point, $u(N)$ is spatial information of Figure 1 transformed into frequency domain using DFT and expressed in Eq. 13. Edge of the boundary in complex form can be given as in Eq. (7)

$$
u(N)=\left(x_{i+1}-x_{i}\right)+j\left(y_{i+1}-y_{i}\right)
$$

Here, centroid distances are computed through the Eq. (8) and the advantage of the said signature is that the Fourier descriptors extracted are robust against noise.

$$
r(t)=\sqrt{\left(x_{t}-x_{c}\right)^{2}+\left(y_{t}-y_{c}\right)^{2}}
$$

where

$$
x_{c}=\sum_{n=0}^{(N-1)} \frac{x(n)}{N} \text { and } y_{c}=\sum_{n=0}^{(N-1)} \frac{y(n)}{N}
$$

and $r(t)$ is the centroid distance which is also called as shape signature and $t=0,1,2 \ldots N-1$ Similarly, literature supports Area Function which can be calculated through the Eq. 10

$$
A(t)=\frac{1}{2}\left[X_{1}(t) Y_{2}(t)-X_{2}(t) Y_{1}(t)\right]
$$

Likewise, another shape quantifier, Curvature function can be estimated through the Eq. 11,

$$
K(t)=\frac{d \theta}{d t}
$$

Where $d t$ is width of the curve and $\theta$ is given by

$$
\theta(t)=\tan ^{-} 1\left(\frac{Y(t)-Y(t-w}{X(t)-X(t-w)}\right)
$$

Where $w$ is step of the curve. The Fourier descriptors of the centroid distance function is estimated through the Eq. [13, then

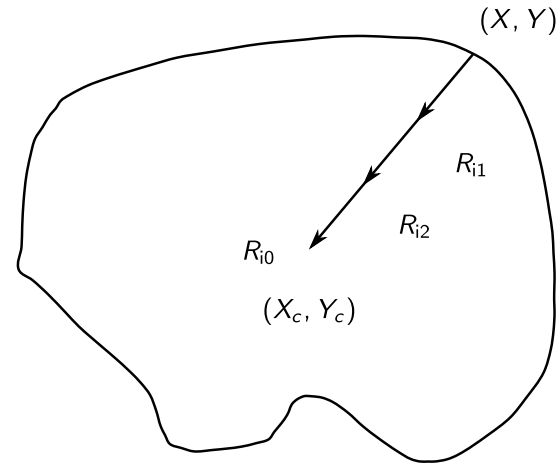

Fig. 2. Geometric mean of segmented centroid distance function.

one dimensional Fourier transform is done on $r(t)$ to get Fourier coefficients $a(n)$

$$
a(n)=\sum_{t=0}^{N-1} r(t) e^{-j 2 \pi n t / N}
$$

where $n=0,1,2 \ldots N-1$. The magnitudes of the Fourier coefficients $a(n)$ are divided by magnitude of first coefficient $a(0)$ to make them invariant to scaling.

$$
\begin{aligned}
& a(n)=\left[\frac{|a(0)|}{|a(0)|}, \frac{|a(1)|}{|a(0)|}, \frac{|a(2)|}{|a(0)|}, \ldots \frac{|a(N-1)|}{|a(0)|}\right] \\
& \mathbf{a}(\mathbf{n})=a(n) e^{-j \phi_{1} n}
\end{aligned}
$$

In order to match between the model template and candidate template to track and update the interested object, a suitable distance measure is considered. Here, the proposed work attempted to employ Minkowski as a proper distance measure.

\subsection{Proposed Shape Signature through the Geometric Mean of Segmented Centroid Distance Function}

The centroid distance function is as given in Eqs. (8) and (9). Here, the referred distance is partitioned into the required $n$ parts as given in Figure 2 through the Eqs. (16)-(17). Segmented centroid distances are calculated through the Eqs. (18)-(21). Geometric mean of the segmented centroid distances for each centroid function $R D$ is estimated via the Eq. 22. Therefore, the vector of the shape signature $R T$ is as per the Eq. 23.

$$
\begin{aligned}
X_{\text {dist }} & =\left(\frac{x_{i}-x_{c}}{n}\right) \\
Y_{\text {dist }} & =\left(\frac{y_{i}-y_{c}}{n}\right)
\end{aligned}
$$

Where $n$ is number of segments on the centroid distance function and $R$ is the absolute value of the $n$-th segment.

$$
\begin{aligned}
R_{i 0} & =\sqrt{\left(x_{i}-x_{c}\right)^{2}+\left(y_{i}-y_{c}\right)^{2}} \\
R_{i 1} & =\sqrt{\left(x_{i}-x_{d i s t}\right)^{2}+\left(y_{i}-y_{d i s t}\right)^{2}} \\
R_{i 2} & =\sqrt{\left(x_{i}-2 x_{d i s t}\right)^{2}+\left(y_{i}-2 y_{d i s t}\right)^{2}} \\
R_{i n} & =\sqrt{\left(x_{i}-n x_{d i s t}\right)^{2}+\left(y_{i}-n y_{d i s t}\right)^{2}} \\
R D_{i} & =\sqrt[n]{R_{i 0} \times R_{i 2} \times R_{i 3} \times \ldots R_{i n}}
\end{aligned}
$$




$$
R T=\left[\begin{array}{c}
R D_{1} \\
R D_{2} \\
R D_{3} \\
\vdots \\
R D_{n}
\end{array}\right]
$$

With the help of Eq.13 Fourier descriptors are enumerated making invariant to scaling, translation, rotation and starting point.

\subsection{Proposed Algorithm}

(1) Convert the image into gray to label as Previous frames.

(2) Read next frame and convert into gray to label as Current frame.

(3) Reckon optical flow via threshold between current frame and previous frame. Also replace current frame as previous frame.

(4) Morphological operations help to get moving objects to form knowledge base.

(5) Testing will be done with object boundary.

(6) Shape signatures are created with Geometric Mean of Segmented Centroid (GMSC) distance Function using Eqs. (16) to 23.

(7) Fourier Descriptors (FDs) are computed for template and moving objects using GMSC.

(8) Similarity is computed between FDs of template and moving objects.

(9) Bounding box is enclosed over the moving object with spatial information corresponding to minimum value of matching. Subsequently, an old template is updated with new one.

(10) Repeat Steps 2 to 9 if video is not ended.

\section{EXPERIMENTS AND RESULTS}

The proposed work has extended the some of the major yardsticks to assess the performance of object tracking algorithm. The parameters such as qualitative, quantitative and time analysis are dominant and potential benchmarks to foretaste the performance evaluation which are comprehensively occupied by respective research inclination. A process of displaying to track the detected objects is emphasized in qualitative analysis. Quantitative analysis has two important metrics that are Detection Rate (DR) and False Alarm Rate (FAR) which are exploited for quantification of proposed strategys outcome as given through Eqs 24) and 25. Using DR and FAR the Combined Result (CR) can be computed as given in Eq. 26. In True Positive (TP) case moving objects and detected regions are identical. On the converse, it belongs to False Positive (FP). In neither of the cases, it fits in False Negative (FN) or misses. The proposed criteria endeavored to exhibit the suitable results, which are tabulated in Table 1 and Figures 3 and 5 The computational aspects of the evolved method turn out to be polynomial and its order is $O\left(n^{3}\right)$. The same is tested over the available machine Pentium(R) Dual-core CPU, T4200 @ 2.00 $\mathrm{GHz}$ and $2.83 \mathrm{~GB}$ of RAM of $1.20 \mathrm{GHz}$.

$$
\begin{aligned}
D R & =\frac{T P}{T P+F N} \times 100 \\
F A R & =\frac{F P}{T P+F P} \times 100 \\
C R & =\frac{D R}{D R+F A R} \times 100
\end{aligned}
$$

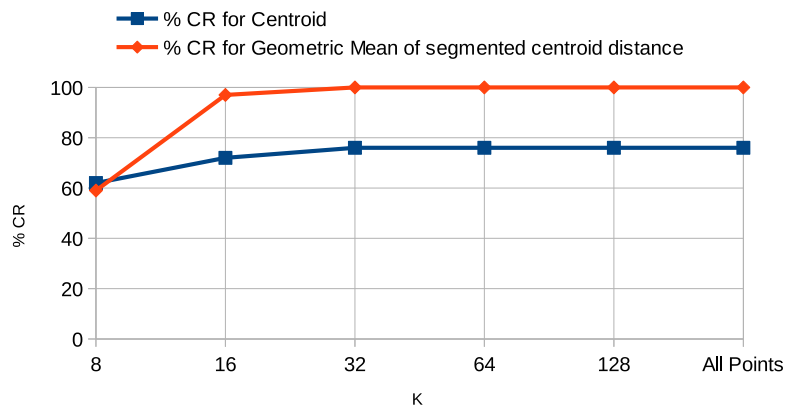

Fig. 3. Comparative results of 29 frames for conventional centroid distance and proposed shape signature of geometric mean of segmented centroid distance function (BC-Black Car-Template-T1, WC-White Car-Template-T2 and obtained FN as 0 and DR as $100 \%$ )

In the first instance, Fourier descriptors values made invariant to translation, scaling, rotation and starting point as depicted in Table 1.

The following are the empirical inferences that are erected as experimental evidences. From the Table 1 complex values for both boundary and centroid distance functions yield similar tracking characteristics. The tracking result of the centroid function as shape signature in absolute form is unsurpassed and it exhibits noticeable performance in tracking with combined result (CR) of $76 \%$. Hence, it can be analyzed that the centroid distance function is robust in tracking the object.

In the second instance centroid distance signature alone has been considered making invariant to various parameters such as FN, FP, FAR and CR. On the converse, the experiment continued on same centroid distance function for non-Invariance.

In both of the cases, boundary points are made variable. However, variability of resolute points which are ambassadors of the shape, are larger in number to contemplate the completeness. The predominant boundary points can also be considered in a sub sampled way by varying the interval size $K$. In view of revealing the strength of the proposed criteria by judiciously quantifying the different value of the $K$ such as $8,16,32,64,128$ and all points. In a simpler way to illustrate the variance of $K, 64$ coordinates are obtained due to value of $K$. The tracking results of the centroid distance function of absolute form is admirable for all $K$ values except for 8 and 16. On the contrary, the tracking results of the centroid distance function of complex form is better for the 8 and 16.

The centroid distance partitioned into 2 segments gave the best result as shown in Figure 4 The results are robust and seen drastic change in the tracking rate of CR as $100 \%$ and $97 \%$ for respective templates. The number of boundary points considered $K$ is also varied from $128,64,32,8$ and 4 . Therefore, it can be comprehended here that using $K$ as 32 alone is sufficient as it gives same results. So the computational burden is reduced but proposed shape signature of GMSC distance works even with lesser $K$. An experiment on the proposed shape signature of GMSC distance has been explored and the results are compared with the conventional centroid function for both dataset. From Figure 5. 
Table 1. Comparative results of Boundary, Edge, and Centroid distance functions - Invariant (obtained FN as 0 and DR as $100 \%$ )

\begin{tabular}{|c|c|c|c|c|c|c|}
\hline Sl. No. & Signature & Signature Type & TP & FP & FAR & CR \\
\hline \multirow{2}{*}{1} & \multirow{2}{*}{ Boundary } & Complex & 06 & 23 & 79 & 56 \\
\hline & & Absolute & 1 & 28 & 97 & 51 \\
\hline \multirow{2}{*}{2} & \multirow{2}{*}{ Edge } & Complex & 06 & 23 & 79 & 56 \\
\hline & & Absolute & 11 & 18 & 62 & 62 \\
\hline \multirow{2}{*}{3} & \multirow{2}{*}{ Area } & Complex & 01 & 28 & 97 & 51 \\
\hline & & Absolute & 01 & 28 & 97 & 51 \\
\hline \multirow{2}{*}{4} & \multirow{2}{*}{ Curvature } & Complex & 12 & 17 & 59 & 63 \\
\hline & & Absolute & 12 & 17 & 59 & 63 \\
\hline \multirow{2}{*}{5} & \multirow{2}{*}{ Centroid } & Complex & 06 & 23 & 79 & 56 \\
\hline & & Absolute & 20 & 09 & 31 & 76 \\
\hline
\end{tabular}

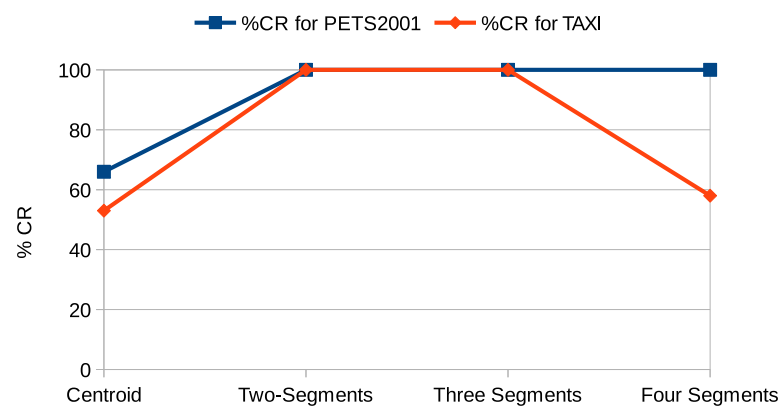

Fig. 4. Comparative results of conventional centroid distance and proposed shape signature of geometric mean of segmented centroid distance function for PETS2001 and TAXI dataset (obtained FN as 0 and DR as 100 $\%)$

it can be observed that the geometric mean distance of the two segments and three segments are excellent.

The value of $K$ as 32 found emperically ideal and hence experimentation has further been continued with proposed geometric mean of segmented centroid distance shape signature on the benchmark dataset such PETS2001 and TAXI. The proposed method exhibited higher performance compared with the existing shape signatures and same may be illustrated through the Figure 5 Samples of tracked frames on PETS2001 for frames (2559-2589) and TAXI dataset are portrayed Figures 6 and 7 respectively.

\section{CONCLUSION}

In this work, Optical flow based Horn-Schunk with BFB kernel has been exercised to contract moving objects. The boundaries of all moving objects are extracted through Canny edge detector due its incomparability among several edge detection principles. Fourier descriptors of the boundaries are prepared as they have advantages like robustness to noise, simple to estimate and compact in size. Fourier descriptors are derived from different shape signatures such as boundary, edge, area, curvature and centroid distance functions. In order to match the template and candidate templates, Minkowski distance has been employed.

Subsequently, centroid distance function is the best shape signature [12] and has assured the robustness. Thus it persuaded to intend the novel GMSC distance function to track the object. An experimental

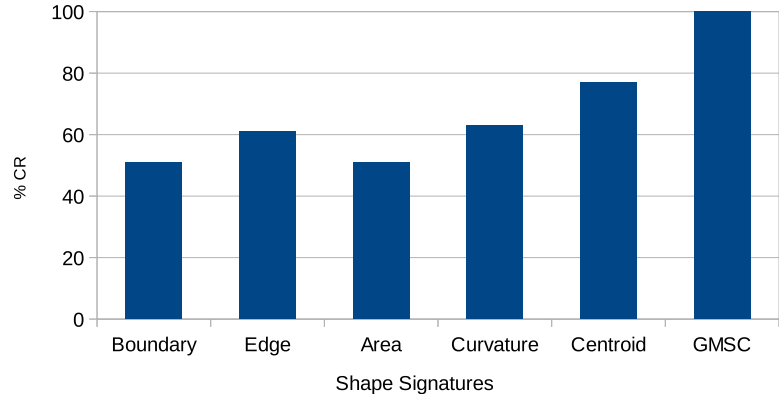

Fig. 5. Comparative Results of absolute values of Various functions and Proposed method)

result on the offered method of GMSC distance function has shown significant change in the tracking results of CR as $100 \%$. Hence, the proposed tracking algorithm has enhanced the robustness against the change in the shape. It is capable of tracking the multiple objects and also can track the smallest object of the size of 50 pixels.

\section{REFERENCES}

[1] Yilmaz A., Javed O., and Shah M. Object tracking: A survey. ACM Computing Surveys, 38,4 Article 13, Dec 2006.

[2] Andre Folkers and Hana Samet. Content based image retrieval using fourier descriptors on a logo database. In Vol. 3, Proceedings of 16th Int.Conf. on Pattern Recognition, pages 521-524, August 2002.

[3] Heesch D. and Ruger S. Combining features for contentbased sketch retrieval a comparative evaluation of retrieval performance. In 24th BCS-IRSG European Colloquium on IR Research, Lecture Notes in Computer Science 2291, pages 41-52. Springer, 2002.

[4] Barron J., Fleet D., and Beauchemin S. Performance of optical flow techniques. Int. J. Comput. Vis., 12(2):43-77, 1994.

[5] Horn B. and Schunck B. Determining optical flow. Artificial Intelligence, 17:185-203, 1981.

[6] Yang Mingqiang, Kpalma Kidiyo, and Ronsin Joseph. A survey of shape feature extraction techniques. Pattern 


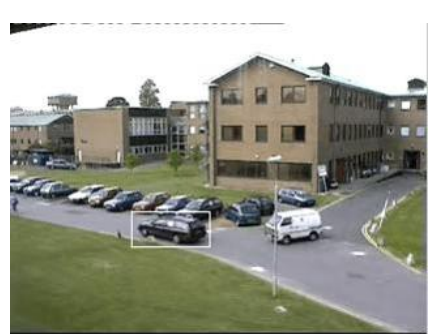

Frame 2559

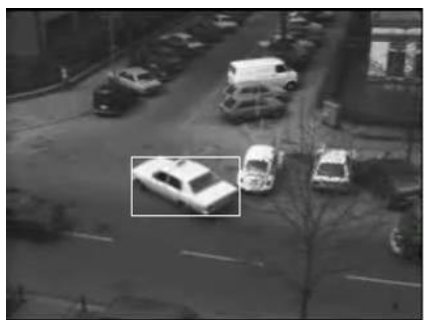

Frame 5

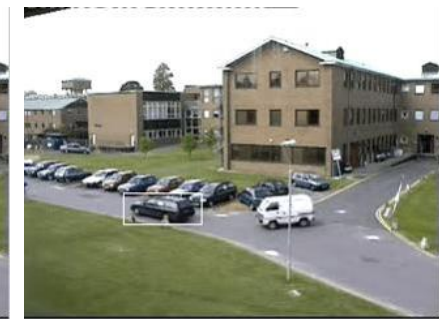

Frame 2568

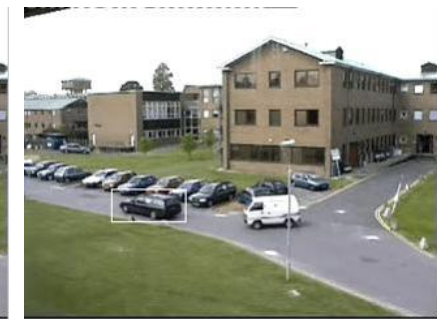

Frame 2578

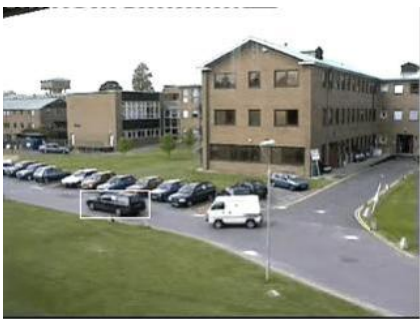

Frame 2588

Fig. 6. PETS2001.

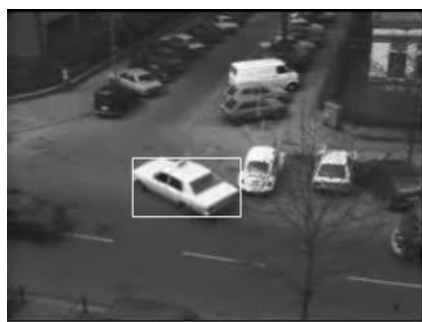

Frame 9

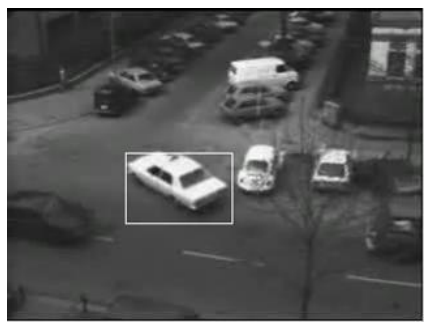

Frame 14

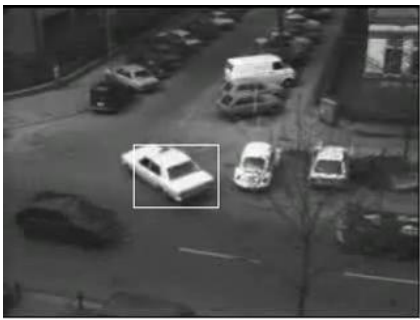

Frame 19

Fig. 7. TAXI.

Recognition Techniques: Technology and Applications, 17:43-90, 2008.

[7] Zhang D. and Lu G. Review of shape representation and description techniques. Pattern Recognition, 37:1-19, 2004.

[8] Zhang D. and Lu G. Shape-based image retrieval using generic fourier descriptor. Signal Processing: Image Communication, 17(10):825-848, 2002.

[9] Zahn C. T. and Roskies R. Z. Fourier descriptors for plane closed curves. IEEE Trans. on Computer Science, C-21(3):269-281, 1972.

[10] Mehtre B M., Kankanhalli M. S., and Lee W. F. Shape measures for content based image retrieval: A comparison. Information Processing and Management, 33(3):319-337, 1997.

[11] Zhang D. and Lu G. Content-based shape retrieval using different shape descriptors: A comparative study. In Proc. of IEEE International Conference on Multimedia and Expo, pages 317-320, August 22-25 2001.

[12] Zhang D. and Lu G. A comparative study of fourier descriptors for shape representation and retrieval. In Proc. 5th Asian Conference on Computer Vision, 2002.

[13] Borel R. J. A mathematical pattern recognition technique-based on contour shape properties. 1965.

[14] Persoon E. and Fu K. Shape discrimination using fourier descriptors. IEEE Trans. on Systems, Man and Cybernetics, 7(3):170-179, 1977.

[15] Amanatiadis A., Kaburlasos V.G., Gasteratos A., and Papadakis S.E. A comparative study of invariant descriptors for shape retrieval. May 11-12 2009.

[16] Belongie S., Malik J., and Puzicha J. Shape matching and object recognition using shape contexts. IEEE Trans. on Pattern Anal. Mach. Intell., pages 509-522, 2002.
[17] Sarfraz M., Mehmood ul Hassan, and Iqbal M. Object recognition using fourier descriptors and genetic algorithm. pages 318-323, 2009. 